\title{
Nervous System
}

10.4 Acute Administration of Vitamin C Decreases Sympathetic Nervous Activity and Blood Pressure in Hypertensive Patients

R.M. Bruno (1), E. Daghini (1), L. Ghiadoni (1), I. Sudano (2), D. Versari (1),

E. Duranti (1), A. Favilla (1), A. Magagna (1), S. Taddei (1), A. Salvetti (1)

(1)Università di Pisa, Pisa, Italy, (2)University Hospital, Zurich, Switzerland

Introduction. Essential hypertension is characterized by both increased oxidative stress and sympathetic nervous system (SNS) hyperactivity. Experimental studies suggest that increased oxidative stress contributes to the development of hypertension through activation of the SNS.

Aim. To determine whether an acute administration of the antioxidant vitamin C can influence SNS activity in hypertensive patients.

Methods. Fifteen never treated, essential hypertensive patients were included. Heart rate (HR), noninvasive beat-to-beat blood pressure (BP, Portapres) and muscle sympathetic nervous activity (mSNA, microneurography) were monitored in basal conditions and until 20 minutes after infusion of vitamin $\mathrm{C}$ ( $3 \mathrm{~g}$ iv in 5 minutes). Venous samples for assessment of norepinephrine (NE), oxidative stress (maloniydialdehyde, MDA) and antioxidant capacity (ferric reducing ability of plasma, FRAP) were obtained before and at the end of recording. HR, BP and mSNA values were analyzed as means of 5 minutes at baseline and between 16'-20' after infusion and expressed as mean \pm SEM.

Results. Vitamin C infusion significantly reduced oxidative stress (MDA from $5.4 \pm 0.8$ to $4.6 \pm 0.6$ micromol/L, $\mathrm{p}<0.05$ ) increased plasma antioxidant capacity (FRAP from $838 \pm 88$ to $2531 \pm 230$ micromol/L, $\mathrm{p}<0.05)$. Moreover, vitamin $\mathrm{C}$ infusion was associated with a significant reduction in $\mathrm{BP}$ (systolic BP from $142.1 \pm 5.2$ to $138.0 \pm 5.7 \mathrm{mmHg}, \mathrm{p}<0.05,-3.0 \%$; diastolic BP from $92.4 \pm 3.3$ to $85.6 \pm 3.4 \mathrm{mmHg}, \mathrm{p}<0.01,-7.0 \%$; mean $\mathrm{BP}$ from $110.5 \pm 3.8$ to $104.6 \pm 4.1 \mathrm{mmHg}, \mathrm{p}=0.01,-5.3 \%$ ) mSNA was significantly reduced (from $32.9 \pm 2.9$ to $29.4 \pm 2.8$ bursts $/ \mathrm{min}, \mathrm{p}=0.01,-10.7 \%$; from $52.1 \pm 3.6$ to $46.3 \pm 3.7$ bursts $/ 100$ heart beats, $\mathrm{p}<0.01,-11.3 \%$ ). NE and HR were unchanged.

Conclusions. Acute administration of vitamin $\mathrm{C}$ is associated with reduced BP and mSNA in hypertensive patients. These preliminary results suggest that oxidative stress may influence the sympathetic hyperactivity characterizing essential hypertension. 